\title{
New combined method for solving the single level capacitated production planning model with set up cost, finite horizon and discrete stochastic demand
}

\author{
Seyed Saeid Hashemin ${ }^{1, *}$, Elham Mohammadi ${ }^{2}$ \\ ${ }^{1}$ Department of Industrial Engineering, Ardabil Branch, Islamic Azad University, Ardabil, Iran \\ ${ }^{2}$ Graduate Student in Industrial Engineering, Ministry of Science, Research and Technology ALGHADIR Non-Governmental and Private \\ Higher Education Institution, Tabriz, Iran
}

Email address:

s.s.hashemin@gmail.com (S. S. Hashemin),Elham.mohammadi64@gmail.com (E. Mohammadi)

\section{To cite this article:}

Seyed Saeid Hashemin, Elham Mohammadi. New Combined Method for Solving the Single Level Capacitated Production Planning Model with Set up Cost, Finite Horizon and Discrete Stochastic Demand. International Journal of Economics, Finance and Management Sciences. Vol. 2, No. 3, 2014, pp. 227-230. doi: 10.11648/j.ijefm.20140203.14

\begin{abstract}
This paper studies the single level capacitated production planning problem with finite horizon ( $\mathrm{N}$ periods). In each period, Set-up cost, variable cost and inventory cost exist. Also, it is assumed that the demand in each period is a discrete random variable with known probability function. In each period, if demand is bigger than inventory then we will have lost sales. In this case, we have to pay the cost of lost sales otherwise at the end of the period we will have extra products for the next period. At the end of horizon we have to sale the surplus products. In this case, price of one unit of products will be less than variable cost of production. An analytical method is proposed for solving this problem. This method can optimize the expected value of costs. In this method, expected value of costs is estimated by Monte Carlo simulation. Two examples have solved by using the proposed method. Comparison of the answers with solutions of other heuristic methods indicates the advantage of the proposed method.
\end{abstract}

Keywords: Capacitated Production Planning, Stochastic Demand, Set Up Cost, Finite Horizon

\section{Introduction}

The literature in production planning (PP) under uncertainty is vast. The problems can be categorized into two groups: (i) environmental uncertainty and (ii) system uncertainty. Uncertainty in this paper lies in group (i). [10] Reviewed the models of PP under uncertainty. [4], [6] considered the stochastic PP when the horizon planning is infinite. [7] Modeled the forecasts of discrete demand as bands and defined them by lower and upper bounds on demand. [1] Proposed a deterministic approximation for the sequential stochastic PP without setup costs. [5] Presented a multi-period hierarchical PP model with two planning levels, i.e. aggregate and detailed, and with uncertain demand. [9] Developed a multi-period model for hierarchical PP and scheduling with random demand and production failure. [8] Studied the capacitated PP with stochastic seasonal demand. [2] Studied the capacity planning under demand uncertainty without setup cost when the plant capacity is flexible. However, this paper studies the single level capacitated PP problem with finite horizon, stochastic demand and set-up cost. In section 2, problem and its mathematical model have been described. In section 3, two present methods have been introduced [11]. Both methods are heuristic. They solve the problem by transforming the stochastic problem to deterministic problem. Then deterministic problems are solved based on an analytical method that has been developed in [3]. A new combined method is developed in section 4. In this method an analytical method has combined with Monte Carlo simulation. Advantage of the new proposed method has been shown by solving two examples in section 5. Section 6 has been devoted to conclusions and recommendations.

\section{Problem and Its Mathematical Model}

Here, we have a single level PP problem with finite horizon. This horizon comprises $\mathrm{N}$ periods. In each period, 
Set-up cost, variable cost and inventory cost exist. Demand in each period is a discrete random variable with known probability function. Production in each period is limited to the production capacity. So, in each period if demand is bigger than the inventory then we will have cost of lost sales. Also, at the end of horizon we have to sale the surplus products. Subject to these suppositions, minimizing the expected value of costs is vital. The mathematical model of problem is stochastic integer linear programming. Some of the variables of model are zero or one and other variables may be continuous or discrete.

This model is as below:

$$
\begin{gathered}
\text { Min } Z=E\left[\sum _ { i = 1 } ^ { N } \left(f_{i} z_{i}+p_{i} x_{i}+h_{i} y_{i}\right.\right. \\
\left.+c_{i} \operatorname{Max}\left(0, D_{i}-x_{i}\right)+\left(\sum_{i=1}^{N} x_{i}-\sum_{i=1}^{N} D_{i}\right) s_{N}\right] \\
\text { s.t. } \quad y_{0}=y_{N}=0 \\
x_{i}+y_{i-1}-y_{i}=D_{i} \\
x_{i}-c_{i} z_{i} \leq 0 \quad z_{i} \in\{0,1\} \\
x_{i} \geq 0, y_{i} \geq 0 \\
i=1,2, \ldots, N
\end{gathered}
$$

where

$D_{i}=$ discrete random variable of demand in period $i$

$p_{i}=$ unit variable production cost in period $i$

$f_{i}=$ set up cost in period $i$

$h_{i}=$ unit holding cost in period $i$

$c_{i}=$ production capacity in period $i$

$N=$ number of periods of planning horizon

$c c_{i}=$ unit cost of lost sale

$S_{N}=$ price of one product at the end of horizon

The model variables are as below:

$x_{i}=$ production quantity in period $i$

$y_{i}=$ inventory at the end of period $i$

$z_{i}=0$ if we do not produce in period $i$

$z_{i}=1$ if we produce in period $i$

\section{Present Methods}

One of the simple ways to solve the stochastic problems is transforming them to deterministic problems. This action can be done by replacing demand with the average of demand. This method is proposed by Hashemin [11]. In this case, objective function can be written as:

$$
\operatorname{Min} \sum_{i=1}^{N}\left(f_{i} z_{i}+p_{i} x_{i}+h_{i} y_{i}\right)
$$

So, in this deterministic model, we have not lost sales.
Also we will not have surplus products at the end of planning horizon. Then, the above mentioned model can be solved with the method proposed by Fatemi Ghomi and Hashemin [3]. Here, this method will be called the first method of reference [11].

Hashemin [11] has proposed a better value to be replaced with demand average. If $c c_{i}$ is big and $h_{i}$ is small then, we prefer that $D_{i}$ to be replaced with value that is larger than $\bar{D}_{i}$. Also, if $c c_{i}$ is small and $h_{i}$ is big then, we prefer that $D_{i}$ to be replaced with value that is smaller than $\bar{D}_{i}$. In other words, this value must be defined by flexible relation. This suitable value $\left(\hat{D}_{i}\right)$ is value that minimized the

$$
\begin{aligned}
& E\left[\operatorname{Max}\left\{0, x_{i}-D_{i}\right\}\left[(N-i+1) h_{i}+p_{i}-s_{N}\right]\right] \\
& +E\left[\operatorname{Max}\left\{D_{i}-x_{i}, 0\right\} c c_{i}\right]
\end{aligned}
$$

Such that $\operatorname{Min}\left\{D_{i}\right\} \leq x_{i} \leq \operatorname{Max}\left\{D_{i}\right\}$ and $x_{i}$ has integer value.

Then, this model can be solved with the method proposed by Fatemi Ghomi and Hashemin [3] too. Here, this method is called the second method of reference [11].

Here for completing the description of present methods, we discuss the proposed method by Fatemi Ghomi and Hashemin [3]. In this model, it is assumed that demand is deterministic in each period and back ordering is not permitted. In many PP problems, variables $x_{i}$ may be integer. It is evident that solving these problems is difficult because the mathematical model of these problems is integer linear programming (ILP) with zero-one variables. Consequently, another analytical method is proposed. The feasible sets $\left\{z_{1}, z_{2}, \ldots, z_{N}\right\}$ can be recognized. It should be noted that there must be $c_{1} \geq D_{1}$ and $z_{1}=1$, in all feasible sets. By replacing the values of $z_{i}$ in mathematical model, it is transformed to a simpler mathematical model. Then, this model must be solved for each feasible set. Implementation of this method can be difficult. Hence, based on reasonable assumptions, an attempt is made to develop a simpler method to solve the sub-problems. These assumptions which may exist in many problems are as follows:

I. The same variable production cost for all periods.

II. The same holding inventory cost for all periods.

Under the above assumptions, the objective function of the problem would be as follows:

$$
\operatorname{Min}\left(\sum_{i=1}^{N} f_{i} z_{i}+p \sum_{i=1}^{N} x_{i}+h \sum_{i=1}^{N} y_{i}\right)
$$

Because the set of values $\left\{z_{1}, z_{2}, \ldots, z_{N}\right\}$ is known for each feasible production plan, then $\sum_{i=1}^{N} f_{i} z_{i}$ is constant for each production plan. Also, because the equivalence 
$y_{0}=y_{N}=0$ exists, there would be $\sum_{i=1}^{N} x_{i}=\sum_{i=1}^{N} D_{i}$. Hence, the next term of objective function, $p \sum_{i=1}^{N} x_{i}$, would also be constant; and $\sum_{i=1}^{N} y_{i}$ should be minimized for each feasible production plan. To do this task, the constraints are divided into two following groups:

$$
\forall z_{i}=1
$$

Group 1: $\quad x_{i}+y_{i-1}-y_{i}=D_{i}$

$$
x_{i} \leq c_{i}
$$$$
\forall z_{i}=0
$$

Group 2: $\quad y_{i-1}-y_{i}=D_{i}$

$$
x_{i}=0
$$

Therefore, if $z_{N}=1$ then, $x_{N}=\operatorname{Min}\left\{c_{N}, D_{N}\right\}$, $y_{N-1}=\operatorname{Max}\left\{0, D_{N}-c_{N}\right\}$ and if $z_{N}=0$, then $y_{N-1}=D_{N}$.

The $(N-1)$ th constraint belongs to either group 1 constraints or group 2 constraints:

I) If $(N-1)$ th constraint belongs to group 1's, then $x_{N-1}+y_{N-2}-y_{N-1}=D_{N-1} \cdot y_{N-1}$ and $D_{N-1}$ are Known; so the value of $x_{N}$ can be determined such that $y_{N-2}$ be minimized. So, $x_{N-1}=\operatorname{Min}\left\{D_{N-1}+y_{N-1}, c_{N-1}\right\} \quad$ and $y_{N-2}=\operatorname{Max}\left\{0, D_{N-1}+y_{N-1}-c_{N-1}\right\}$.In general if $z_{i}=1$ then, $y_{i-1}=\operatorname{Max}\left\{0, D_{i}+y_{i}-c_{i}\right\}$.

II) If $(N-1)$ th constraint belongs to group 2's, then $y_{N-2}=D_{N-1}+y_{N-1}$. In general, if $z_{i}=0$, then $y_{i-1}=D_{i}+y_{i}$.

Repeating the above operations for all periods gives the values of $x_{i}$ and $y_{i}$.

\section{Proposed Method}

Proposed method generates the values of random variable $D_{i}, i=1,2, \ldots, N$. Then, by replacing $D_{i}$ with these values, the obtained model, is solved by using the method proposed by Fatemi Ghomi and Hashemin [3]. So, values of $x_{i}, i=1,2, \ldots, N$ are defined. For each solved model (in other words, for each set of obtained $\left.x_{i}, i=1,2, \ldots, N\right)$ we generate the values of random variable $D_{i}, i=1,2, \ldots, N$ again. Then, we can compute the expected value of total costs. Finally, $x_{i}, i=1,2, \ldots, N$ which has the minimum expected value of total costs are introduced as the best solution.

For implementation of proposed method, an algorithm is developed as below:

It is assumed that number of all cases of demand values of periods is $M$. Also, suppose that the primal value of $M E T C$ is $+\infty$. In this algorithm METC will show the
Minimum Expected Value of Total Cost.

Step 1: set $L=1$.

Step 2: generate the values of random variable $D_{i}, i=1,2, \ldots, N$.

Step 3: by replacing $D_{i}$ with these values, solve the obtained model by using the method proposed by Fatemi Ghomi and Hashemin [3] and define the values of $x_{i}, i=1,2, \ldots, N$.

Step 4: compute the expected value of total cost and set $E T C=$ The expected value of total cost.

Step 5: if $E T C<M E T C$ then set $M E T C=E T C$.

Step 6: if $L=M$ stop. In this case value of $M E T C$ is the minimum value for expected value of total cost and values of $x_{i}, i=1,2, \ldots, N$ is optimum otherwise set $L=L+1$ and generate the values of random variable $D_{i}, i=1,2, \ldots, N$ again (other case). Then go to step 3 .

If the size of problem is small we can generate all the value of random variable $D_{i}, i=1,2, \ldots, N$. Therefore we can obtain the optimum solutions. In large scale problems, these values can be generated by Monte Carlo simulation. In this case, we can't make sure that solutions are optimum but we know that by increasing the number of simulation runs we can obtain better solutions.

\section{Examples}

Example 1: consider the problem with

$$
\begin{gathered}
f_{i}=2.5 \quad P_{i}=4 \quad h_{i}=0.3 \quad c c_{i}=1.5 \\
c_{i}=15 \quad s_{6}=1 \quad i=1,2, \ldots, 6
\end{gathered}
$$

Probability functions of demands in six periods are shown in Table 1 and Table 2.

Table 1. Probability Functions of Periods 1,2,3 in Example 1

\begin{tabular}{llllll}
\hline$D_{1}$ & $P\left(D_{1}\right)$ & $D_{2}$ & $P\left(D_{2}\right)$ & $D_{3}$ & $P\left(D_{3}\right)$ \\
\hline 6 & 0.175 & 4 & 0.1 & 2 & 0.15 \\
7 & 0.175 & 5 & 0.3 & 3 & 0.2 \\
8 & 0.3 & 6 & 0.3 & 4 & 0.3 \\
9 & 0.175 & 7 & 0.1 & 5 & 0.2 \\
10 & 0.175 & 8 & 0.2 & 6 & 0.15 \\
\hline
\end{tabular}

Table 2. Probability Functions of Periods 4,5,6 in Example 1

\begin{tabular}{llllll}
\hline$D_{4}$ & $P\left(D_{4}\right)$ & $D_{5}$ & $P\left(D_{5}\right)$ & $D_{6}$ & $P\left(D_{6}\right)$ \\
\hline 9 & 0.1875 & 5 & 0.15 & 3 & 0.2 \\
10 & 0.1875 & 6 & 0.2 & 4 & 0.2 \\
11 & 0.25 & 7 & 0.3 & 5 & 0.2 \\
12 & 0.1875 & 8 & 0.2 & 6 & 0.2 \\
13 & 0.1875 & 9 & 0.15 & 7 & 0.2 \\
\hline
\end{tabular}

Solutions of present methods are compared with solution of proposed method in Table 3. 
Table 3. Solutions of present methods and proposed method in example 1

\begin{tabular}{lllllllll}
\hline Methods & $i$ & $\mathbf{1}$ & $\mathbf{2}$ & $\mathbf{3}$ & $\mathbf{4}$ & $\mathbf{5}$ & $\mathbf{6}$ & $\begin{array}{l}\text { Expected } \\
\text { value of Total } \\
\text { Cost }\end{array}$ \\
\hline First & $\bar{D}_{i}$ & 8 & 6 & 4 & 11 & 7 & 0 & 185.1845 \\
Method & $X_{i}$ & 8 & 10 & 0 & 11 & 12 & 0 & \\
Second & $\hat{D}_{i}$ & 7 & 5 & 3 & 10 & 6 & 4 & 169.3261 \\
Method & $X_{i}$ & 15 & 0 & 0 & 10 & 10 & 0 & \\
Proposed & $D_{i}$ & 6 & 4 & 2 & 9 & 5 & 5 & 148.9991 \\
Method & $X_{i}$ & 12 & 0 & 0 & 9 & 8 & 0 & \\
\hline
\end{tabular}

Example 2: consider the problem with

$$
\begin{gathered}
f_{i}=25 \quad P_{i}=4 \quad h_{i}=0.3 \quad c c_{i}=15 \\
c_{i}=120 \quad \mathrm{~s}_{6}=1 \quad \mathrm{i}=1,2, \ldots, 6
\end{gathered}
$$

Probability functions of demands in six periods are shown in Table 4 and Table 5.

Table 4. Probability Functions of Periods 1,2,3 in Example 2

\begin{tabular}{llllll}
\hline$D_{1}$ & $P\left(D_{1}\right)$ & $D_{2}$ & $P\left(D_{2}\right)$ & $D_{3}$ & $P\left(D_{3}\right)$ \\
\hline 40 & 0.1 & 60 & 0.1 & 20 & 0.1 \\
50 & 0.1 & 70 & 0.1 & 30 & 0.2 \\
60 & 0.1 & 80 & 0.3 & 40 & 0.5 \\
70 & 0.2 & 90 & 0.4 & 50 & 0.1 \\
80 & 0.5 & 100 & 0.1 & 60 & 0.1 \\
\hline
\end{tabular}

Table 5. Probability Functions of Periods 4,5,6 in Example 2

\begin{tabular}{llllll}
\hline$D_{4}$ & $P\left(D_{4}\right)$ & $D_{5}$ & $P\left(D_{5}\right)$ & $D_{6}$ & $P\left(D_{6}\right)$ \\
\hline 80 & 0.1 & 10 & 0.1 & 30 & 0.2 \\
90 & 0.1 & 20 & 0.3 & 40 & 0.4 \\
100 & 0.2 & 30 & 0.4 & 50 & 0.2 \\
110 & 0.5 & 40 & 0.1 & 60 & 0.1 \\
120 & 0.1 & 50 & 0.1 & 70 & 0.1 \\
\hline
\end{tabular}

Solutions of present methods are compared with solution of proposed method in Table 6 .

Table 6. Solutions of present methods and proposed method in example 2

\begin{tabular}{lllllllll}
\hline Methods & $i$ & $\mathbf{1}$ & $\mathbf{2}$ & $\mathbf{3}$ & $\mathbf{4}$ & $\mathbf{5}$ & $\mathbf{6}$ & $\begin{array}{l}\text { Expected } \\
\text { value of } \\
\text { total cost }\end{array}$ \\
\hline $\begin{array}{llllllll}\text { First } \\
\text { Method }\end{array}$ & $\bar{D}_{i}$ & 75 & 83 & 36 & 104 & 28 & 45 & 2406.846 \\
$\begin{array}{l}\text { Second } \\
\text { Method }\end{array}$ & $\hat{D}_{i}$ & 80 & 90 & 40 & 110 & 30 & 50 & 2230.408 \\
& $X_{i}$ & 90 & 120 & 0 & 110 & 80 & 0 & \\
$\begin{array}{l}\text { Proposed } \\
\text { Method }\end{array}$ & $D_{i}$ & 80 & 90 & 50 & 110 & 30 & 40 & \\
\hline
\end{tabular}

\section{Conclusions and Recommendations}

The new proposed method reduces the expected value of total cost. It is evident that in large scale problems by increasing the number of simulation we can obtain better solutions.

Using the proposed method is recommended when the demand of periods are continuous random variables.

Solving the multi level problems with proposed method can be studied in future researches.

\section{References}

[1] G.R. Bitran and H.H. Yanasse, "Deterministic approximations to stochastic production problems," Operations Research, 32(5) (1984), 999-1018.

[2] D. Bose and A.K. Chatterjee, "Capacity planning under demand uncertainty: production postponement and production flexibility," CLAIO 2008, September 9-12, Cartagena de Indias, Colombia.

[3] S.M.T. Fatemi Ghomi and S.S.Hashemin, "An analytical method for single level constrained resources production problem with constant setup cost," Iranian Journal of Science and Technology, Transaction B, 26(B1) (2002), 69-82.

[4] A. Garcia and R.L. Smith, "Solving nonstationary infinite horizon stochastic production planning problems," Operations research letters, 27(2000), 135-141.

[5] H. Gfrerer and G. Zapfel, "Hierarchical model for production planning in the case of uncertain demand," European Journal of Operational, 86(1995), 142-161.

[6] K. Huang and S. Ahmed, "A stochastic programming approach for planning horizons of infinite horizon capacity planning problems," European Journal of Operational Research, 200(1) (2010), 74-84.

[7] P. Kaminsky and J.M. Swaminathan, "Effective heuristics for capacitated production planning with multiperiod production and demand with forecast band refinement," Manufacturing \& Service Operations Management, 6(2) (2004), 184-194.

[8] R. Metters, "Production planning with stochastic seasonal demand and capacitated production," IIE Transactions, 29(11) (1997), 1017-1029.

[9] M.Z. Meybodi and B.L. Foote, "Hierarchical production planning and scheduling with random demand and production failure," Annals of Operations Research, 59(1) (2005), 259-280.

[10] J. Mula, R. Poler, J.P. Garcia-Sabater and F.C. Lario, "Models for production planning under uncertainty: A review," International Journal of Production Economics, 103(2006), 271-285.

[11] S.S. Hashemin, "Heuristic for single level capacitated production planning problem with stochastic demand and constant set-up cost, "The 2nd International Conference of Iranian Operation Research society, may 20-22. 2009. 